\title{
Évolution de la pratique de la rachianesthésie pour les césariennes en Côte d'Ivoire
}

\author{
Edmond Konan Kouamé, PhD • Abdoulaye Ouattara, MD • \\ Démisère Yaïch Peté, MD
}

Received: 22 May 2013/Accepted: 15 July 2013/Published online: 30 July 2013

(C) Canadian Anesthesiologists' Society 2013

\section{Au rédacteur en chef,}

La rachianesthésie est actuellement la technique anesthésique locorégionale qui procure le plus de sécurité materno-fotale et d'analgésie postopératoire. En 2003, la majorité des césariennes réalisées en Côte-d'Ivoire se déroulaient sous anesthésie générale $(87 \%)$ et seulement $13 \%$ sous rachianesthésie. ${ }^{1}$ Depuis ce temps, des activités de formation continue sur l'anesthésie locorégionale (ateliers, séminaires, congrès...) ont été organisées pour les anesthésiologistes, les internes et les infirmiers anesthésistes. Nous voulons ici décrire l'impact de cette promotion de la rachianesthésie pour les césariennes. Nous avons réalisé une étude rétrospective à partir des fiches d'anesthésie et du registre des actes anesthésiques du bloc opératoire obstétrical du Centre hospitalier universitaire (CHU) de Cocody à Abidjan pour les césariennes au cours des trois années s'étalant du 1er janvier 2010 au 31 décembre 2012.

$\mathrm{Au}$ cours de cette période nous avons réalisé 4499 césariennes dont 3866 sous rachianesthésie $(86 \%)$ et les échecs de cette technique étaient de 188 cas $(4,8 \%)$ (Tableau). Dans $90 \%$ des cas, il s'agissait d'interventions en urgence contre $10 \%$ de césariennes programmées. Les patientes appartenaient pour la plupart à la classe ASA I (American Society of Anesthesiologists) (65\%). La souffrance fœtale aigüe était la principale indication de la césarienne $(35,6 \%)$. Le calibre moyen des aiguilles utilisées pour la rachianesthésie était de $26 \mathrm{G}$ et le niveau de ponction était L2-L3 (41,7 \%) ou L3-L4 (58,3\%). L'anesthésique local était la bupivacaïne isobare à une dose moyenne de $10 \pm 0,4 \mathrm{mg}$ et la morphine été l'adjuvant

E. K. Kouamé, PhD · A. Ouattara, MD $(\varangle)$ · D. Y. Peté, MD CHU Cocody, Abidjan, Côte d'Ivoire

e-mail: doc_ablo@yahoo.fr anesthésique. L'hypotension artérielle a été observée en per opératoire chez $57,7 \%$ des patientes et traitée efficacement par le remplissage vasculaire associé à l'éphédrine. Aucun décès maternel n'a été noté. Nous avons enregistré 32 décès de nouveau-nés après extraction ou au décours d'une réanimation néonatale.

Cette étude montre les efforts réalisés en matière de rachianesthésie avec des taux de réalisation proches de ceux des pays d'Europe. ${ }^{2}$ L'accouchement au cours des souffrances fotales aigües impose une extraction urgente du fotus dans un délai de cinq minutes, ce qui n'est réalisable que sous anesthésie générale avec des produits à portée de main. Dans notre contexte, les produits anesthésiques (hypnotiques) ne sont pas toujours disponibles au niveau des pharmacies des hôpitaux. Le recours à la rachianesthésie constitue dès lors une alternative séduisante du fait de la disponibilité dans nos blocs opératoires de la bupivacaïne. Malgré le risque de contamination, il est courant d'utiliser un même flacon de bupivacaïne pour plusieurs (jusqu'à six) anesthésies.

L'hypotension artérielle est une complication fréquente de la rachianesthésie. La phényléphrine, agent alpha-agoniste pur, permet de corriger l'hypotension et minimise la survenue d'une acidose fœtale avec moins d'effets secondaires que l'éphédrine seule. ${ }^{3}$ Malheureusement nous ne disposons pas à ce jour de cette molécule dans nos blocs opératoires. Les nouveau-nés étaient évalués uniquement à l'aide du score d'Apgar, l'analyse du $\mathrm{pH}$ du cordon n'étant pas disponible dans notre établissement.

En dépit d'une mortinatalité et d'une mortalité néonatale précoce élevées sur la période de l'étude, la promotion de la rachianesthésie a permis de réduire la mortalité maternelle. Son faible coût, sa facilité de réalisation et ses risques moindres font d'elle une anesthésie à 
Tableau Césariennes sous rachianesthésie au cours des années 2003, 2010, 2011 et 2012

\begin{tabular}{lllll}
\hline Variables & Période & & & \\
\cline { 2 - 5 } & 2003 & 2010 & 2011 & 2012 \\
\hline Césariennes $(n)$ & 937 & 1366 & 1336 & 1797 \\
Rachianesthésie $(n$ et $\%)$ & $122(13 \%)$ & $1136(83 \%)$ & $1149(86 \%)$ & $1581(87 \%)$ \\
Nombre d'échecs à la rachianesthésie & 59 & 64 & 53 & 71 \\
\hline
\end{tabular}

encourager et à promouvoir s'il n'existe pas de contre-indication absolue dans nos hôpitaux peu équipés.

\section{Remerciements au professeur Emmanuel Bissagnéné.}

\section{Références}

1. Tetchi $Y$, Anoh N'D, N'guessan YF, Ouattara A, Brouh $Y$, Mignonsin D. Pratique anesthésique en chirurgie d'urgence:
Analyse de deux années de pratique au CHU de Cocody à Abidjan en Côte d'Ivoire. Rev Afr Anesth Med Urg 2006; 11: 15-20.

2. Tsen LC, Pitner R, Camann WR. General anesthesia for cesarean section at a tertiary care hospital 1990-1995: indications and applications. Int J Obstet Anesth 1998; 7: 147-52.

3. Mercier FJ, Bonnet MP, De la Dorie A, et al. Rachianesthésie pour césarienne: remplissage, vasopresseurs et hypotension. Ann Fr Anesth Reanim 2007; 26: 688-93. 